\title{
Tate curves and UHF-algebras
}

\author{
Igor Nikolaev
}

\begin{abstract}
It is proved that (a stabilization of) the norm-closure of a selfadjoint representation of the twisted homogeneous coordinate ring of a Tate curve contains a copy of the UHF-algebra.
\end{abstract}

Key words and phrases: Tate curves, UHF-algebras

MSC: $11 G 07$ (elliptic curves over local fields); $46 L 85$ (noncommutative topology)

\section{Introduction}

The Tate curve $\mathcal{E}_{q}$ is an affine cubic over the field $\mathbf{Q}_{p}$ of $p$-adic numbers:

$$
y^{2}+x y=x^{3}-\left(5 \sum_{n=1}^{\infty} \frac{n^{3} q^{n}}{1-q^{n}}\right) x-\frac{1}{12} \sum_{n=1}^{\infty} \frac{\left(5 n^{3}+7 n^{5}\right) q^{n}}{1-q^{n}},
$$

where $q$ is a $p$-adic integer satisfying condition $0<|q|<1$. If $q^{\mathbb{Z}}=\left\{q^{n}: n \in\right.$ $\mathbb{Z}\}$ is a lattice and $\mathbf{Q}_{p}^{*}$ is the group of units of $\mathbf{Q}_{p}$, then the action $x \mapsto q x$ is discrete; in particular, the quotient $\mathbf{Q}_{p}^{*} / q^{\mathbb{Z}}$ is the Hausdorff topological space. It was proved by Tate, that there exists an (analytic) isomorphism $\phi: \mathbf{Q}_{p}^{*} / q^{\mathbb{Z}} \rightarrow \mathcal{E}_{q}$, [Tate 1974] [12], p.190.

The $U H F$-algebra $M_{\mathbf{k}}$ ("uniformly hyper-finite $C^{*}$-algebra") is an inductive limit of the form:

$$
M_{k_{1}}(\mathbb{C}) \rightarrow M_{k_{1}}(\mathbb{C}) \otimes M_{k_{2}}(\mathbb{C}) \rightarrow M_{k_{1}}(\mathbb{C}) \otimes M_{k_{2}}(\mathbb{C}) \otimes M_{k_{3}}(\mathbb{C}) \rightarrow \ldots,
$$

where $M_{k_{i}}(\mathbb{C})$ is a matrix $C^{*}$-algebra, $k_{i} \in\{1,2,3, \ldots\}$ and $\mathbf{k}=\left(k_{1}, k_{2}, k_{3}, \ldots\right)$ [Glimm 1960] [5]. Recall that the algebras $M_{\mathbf{k}}$ and $M_{\mathbf{k}^{\prime}}$ are said to be stably isomorphic (Morita equivalent) if $M_{\mathbf{k}} \otimes \mathcal{K} \cong M_{\mathbf{k}^{\prime}} \otimes \mathcal{K}$, where $\mathcal{K}$ is the 
$C^{*}$-algebra of compact operators; the $C^{*}$-algebra $M_{\mathbf{k}} \otimes \mathcal{K}$ is called a stabilization of $M_{\mathbf{k}}$. (The Morita equivalence of $M_{\mathbf{k}}$ and $M_{\mathbf{k}^{\prime}}$ means that the corresponding non-commutative topological spaces are homeomorphic.) Glimm classified stable isomorphism classes of the UHF-algebras as follows. Let $p$ be a prime number and $n=\sup \left\{0 \leq j \leq \infty: p^{j} \mid \prod_{i=1}^{\infty} k_{i}\right\}$; denote by $\mathbf{n}=\left(n_{1}, n_{2}, \ldots\right)$ an infinite sequence of $n_{i}$, where $p_{i}$ runs through all primes. By $\mathbb{Q}(\mathbf{n})$ one understands an additive subgroup of $\mathbb{Q}$ consisting of the rational numbers whose denominators divide the "supernatural number" $p_{1}^{n_{1}} p_{2}^{n_{2}} \ldots$, where each $n_{j}$ belongs to the set $\{0,1,2, \ldots, \infty\}$. The $\mathbb{Q}(\mathbf{n})$ is a dense subgroup of $\mathbb{Q}$ and every dense subgroup of $\mathbb{Q}$ containing $\mathbb{Z}$ is given by $\mathbb{Q}(\mathbf{n})$ for some $\mathbf{n}$, see e.g. [Rørdam, Larsen \& Laustsen 2000] [8], Proposition 7.4.3 (i). The UHF-algebra $M_{\mathbf{k}}$ and the group $\mathbb{Q}(\mathbf{n})$ are connected by the formula $K_{0}\left(M_{\mathbf{k}}\right) \cong \mathbb{Q}(\mathbf{n})$, where $K_{0}\left(M_{\mathbf{k}}\right)$ is the $K_{0}$-group of the $C^{*}$-algebra $M_{\mathbf{k}}$. The UHF-algebras $M_{\mathbf{k}}$ and $M_{\mathbf{k}^{\prime}}$ are stably isomorphic if and only if $r \mathbb{Q}(\mathbf{n})=s \mathbb{Q}\left(\mathbf{n}^{\prime}\right)$ for some positive integers $r$ and $s$, see [Effros 1981] [4], p. 28 or [Glimm 1960] [5].

The aim of our note is a link between geometry of the Tate curves $\mathcal{E}_{q}$ and a class of the UHF-algebras $M_{\mathbf{k}}$. Such a link is based on the ideas of noncommutative algebraic geometry; we refer the reader to [Stafford \& van den Bergh 2001] [11] for an excellent introduction to the area. Namely, we consider the closure of a self-adjoint representation of the twisted homogeneous coordinate ring of the Tate curve $\mathcal{E}_{q}$ by the linear operators on a Hilbert space; such a closure is always a $C^{*}$-algebra, see e.g. [Murphy 1990] [7]. An independent study of a related problem has been undertaken by [Carey, Marcolli \& Rennie 2011] [2]. Let us pass to an exact statement of our result.

It is known that each $p$-adic integer $0<|q|<1$ is the limit of a convergent series of the rational integers $\alpha_{k}=\sum_{i=1}^{k} b_{i} p^{i}$, where $0 \leq b_{i} \leq p-1$ and $q=\sum_{i=1}^{\infty} b_{i} p^{i}$, see e.g. [Gouvêa 1993] [6], p. 66 for the details. For each $\alpha_{k}$ one can define a supernatural number $\mathbf{n}\left(\alpha_{k}\right)$ of the form $p_{1}^{\infty} \ldots p_{s}^{\infty}$, where $\mathcal{P}_{k}:=\left\{p_{1}, \ldots, p_{s}\right\}$ the finite set of all primes dividing $\alpha_{k}$. By $M_{\alpha_{k}}$ we shall understand (the stable isomorphism class of) an UHF-algebra, such that $K_{0}\left(M_{\alpha_{k}}\right) \cong \mathbb{Q}\left(\mathbf{n}\left(\alpha_{k}\right)\right)$. Let $\left\{\pi_{1}, \pi_{2}, \ldots\right\}$ be a (finite or infinite) set of all primes, such that $\pi_{j} \in \bigcup_{k=1}^{\infty} \mathcal{P}_{k}$. By $\mathbf{n}(q)$ we shall understand a supernatural number of the form $\pi_{1}^{\infty} \pi_{2}^{\infty} \ldots$ and by $M_{q}$ an UHF-algebra, such that $K_{0}(M(q)) \cong \mathbb{Q}(\mathbf{n}(q))$; in other words, $M_{q}$ is the smallest UHF-algebra containing all the UHF-algebras $M_{\alpha_{k}}$. Our main result can be stated as follows. 
Theorem 1 A stabilization of the norm-closure of a self-adjoint representation of the (quotient of) twisted homogeneous coordinate ring of the Tate curve $\mathcal{E}_{q}$ contains a copy the UHF-algebra $M_{q}$.

The note is organized as follows. Section 2 introduces notation and some preliminary facts. Theorem 1 is proved in Section 3; the proof is based on lemma 5. A numerical example illustrating theorem 1 is constructed in Section 4 .

\section{Preliminaries}

This section contains a brief description of the twisted homogeneous coordinate ring of an elliptic curve - the so-called Sklyanin algebra [Sklyanin 1982] [9]; for a general theory of such rings we refer the reader to [Stafford \& van den Bergh 2001] [11. The Cuntz-Krieger $C^{*}$-algebras were introduced in [Cuntz \& Krieger 1980 ] [3]; their $K$-theory and the crossed product structure are discussed in [Blackadar 1986] [1], Exercise 10.11.9. We consider the two-dimensional Cuntz-Krieger algebras only; the latter link the Sklyanin *-algebras with the UHF-algebras.

\subsection{Sklyanin algebras}

Denote by $k$ be a field of characteristic different from two. By a fourdimensional Sklyanin algebra $\mathfrak{S}_{\alpha, \beta, \gamma}(k)$ one understands a free $k$-algebra on four generators $x_{i}$ which satisfy six quadratic relations:

$$
\left\{\begin{array}{l}
x_{1} x_{2}-x_{2} x_{1}=\alpha\left(x_{3} x_{4}+x_{4} x_{3}\right), \\
x_{1} x_{2}+x_{2} x_{1}=x_{3} x_{4}-x_{4} x_{3}, \\
x_{1} x_{3}-x_{3} x_{1}=\beta\left(x_{4} x_{2}+x_{2} x_{4}\right), \\
x_{1} x_{3}+x_{3} x_{1}=x_{4} x_{2}-x_{2} x_{4}, \\
x_{1} x_{4}-x_{4} x_{1}=\gamma\left(x_{2} x_{3}+x_{3} x_{2}\right), \\
x_{1} x_{4}+x_{4} x_{1}=x_{2} x_{3}-x_{3} x_{2},
\end{array}\right.
$$

where $\alpha, \beta, \gamma \in k$ and $\alpha+\beta+\gamma+\alpha \beta \gamma=0$. Assume that $\alpha \notin\{0 ; \pm 1\}$; then algebra $\mathfrak{S}_{\alpha, \beta, \gamma}(k)$ defines a non-singular elliptic curve given as the intersection of two quadrics:

$$
\mathcal{E}(k)=\left\{(u, v, w, z) \in \mathbb{P}^{3}(k) \mid u^{2}+v^{2}+w^{2}+z^{2}=\right.
$$




$$
\left.=\frac{1-\alpha}{1+\beta} v^{2}+\frac{1+\alpha}{1-\gamma} w^{2}+z^{2}=0\right\}
$$

together with an automorphism $\sigma: \mathcal{E}(k) \rightarrow \mathcal{E}(k)$, see [Sklyanin 1982] [9] and [Smith \& Stafford 1992] [10], p. 267. The critical fact that we shall use in the future is the following isomorphism:

$$
\operatorname{QGr}\left(\mathfrak{S}_{\alpha, \beta, \gamma}(k) / \Omega\right) \cong \mathbf{Q} \operatorname{coh}(\mathcal{E}(k)),
$$

where QGr is a category of the quotient graded modules over the algebra $\mathfrak{S}_{\alpha, \beta, \gamma}(k)$ modulo torsion, Qcoh a category of the quasi-coherent sheaves on $\mathcal{E}(k)$ and $\Omega \subset \mathfrak{S}_{\alpha, \beta, \gamma}(k)$ a two-sided ideal generated by the central elements $\Omega_{1}=x_{1}^{2}+x_{2}^{2}+x_{3}^{2}+x_{4}^{2}$ and $\Omega_{2}=x_{2}^{2}+\frac{1+\beta}{1-\gamma} x_{3}^{2}+\frac{1-\beta}{1+\alpha} x_{4}^{2}$, see [Sklyanin 1982] [9], Theorem 2. Since (5) coincides with well-known isomorphism linking projective variety and its homogeneous coordinate ring in the classical (i.e. commutative) algebraic geometry but algebra $\mathfrak{S}_{\alpha, \beta, \gamma}(k)$ is no longer commutative (unless $\sigma$ is trivial), one calls $\mathfrak{S}_{\alpha, \beta, \gamma}(k)$ (modulo an ideal) a twisted homogeneous coordinate ring of elliptic curve $\mathcal{E}(k)$. For a general theory we refer the reader to [Stafford \& van den Bergh 2001] [11].

Lemma 1 For $\beta=1$ and $\gamma=-1$ elliptic curve given by equations (4) is isomorphic to such in the Legendre normal form:

$$
y^{2}=x(x-1)(x-\alpha) .
$$

Proof. For $\beta=1$ and $\gamma=-1$, one can write (4) in the form:

$$
\left\{\begin{array}{ccc}
(1-\alpha) v^{2}+(1+\alpha) w^{2}+2 z^{2} & = & 0 \\
u^{2}+v^{2}+w^{2}+z^{2} & = & 0 .
\end{array}\right.
$$

We shall pass in (77) from variables $(u, v, w, z)$ to the new variables $(X, Y, Z, T)$ given by the formulas

$$
\left\{\begin{array}{ccc}
u^{2} & = & T^{2} \\
v^{2} & = & \frac{1}{2} Y^{2}-\frac{1}{2} Z^{2}-T^{2} \\
w^{2} & = & X^{2}+\frac{1}{2} Y^{2}-\frac{1}{2} Z^{2}-T^{2} \\
z^{2} & = & Z^{2}
\end{array}\right.
$$

Then equations (7) take the form

$$
\left\{\begin{array}{c}
\alpha X^{2}+Z^{2}-T^{2}=0 \\
X^{2}+Y^{2}-T^{2}=0
\end{array}\right.
$$


Let us consider another (polynomial) transformation $(x, y) \mapsto(X, Y, Z, T)$ given by the formulas

$$
\left\{\begin{array}{ccc}
X & = & -2 y \\
Y & = & x^{2}-1+\alpha \\
Z & = & x^{2}+2(1-\alpha) x+1-\alpha \\
T & = & x^{2}+2 x+1-\alpha
\end{array}\right.
$$

Then both of the equations (9) give us the equation $y^{2}=x(x+1)(x+1-\alpha)$, which after a shift $x^{\prime}=x+1$ takes the Legendre form $y^{2}=x(x-1)(x-\alpha)$. Lemma 1 follows.

Corollary 1 Whenever $\beta=1$ and $\gamma=-1$, one can replace in (4) $\alpha$ by $1-\alpha$.

Proof. For elliptic curve $\mathcal{E}_{\alpha}$ in the Legendre form $y^{2}=x(x-1)(x-\alpha)$ the $j$-invariant is given by well-known formula $j\left(\mathcal{E}_{\alpha}\right)=2^{6} \frac{\left(\alpha^{2}-\alpha+1\right)^{3}}{\alpha^{2}(\alpha-1)^{2}}$. It is verified directly, that $j\left(\mathcal{E}_{\alpha}\right)=j\left(\mathcal{E}_{1-\alpha}\right)$, i.e. $\mathcal{E}_{\alpha}$ is isomorphic to $\mathcal{E}_{1-\alpha}$.

\subsection{Cuntz-Krieger algebras}

Let $A$ be a two-by-two matrix with the non-negative integer entries $a_{i j}$, such that every row and every column of $A$ is non-zero. The two-dimensional Cuntz-Krieger algebra $\mathcal{O}_{A}$ is a $C^{*}$-algebra of bounded linear operators on a Hilbert space $\mathcal{H}$, which is generated by the partial isometries $s_{1}$ and $s_{2}$, and relations:

$$
\left\{\begin{array}{ccc}
s_{1}^{*} s_{1} & = & a_{11} s_{1} s_{1}^{*}+a_{12} s_{2} s_{2}^{*}, \\
s_{2}^{*} s_{2} & = & a_{21} s_{1} s_{1}^{*}+a_{22} s_{2} s_{2}^{*}, \\
I d & = & s_{1} s_{1}^{*}+s_{2} s_{2}^{*},
\end{array}\right.
$$

where $I d$ is the identity operator on $\mathcal{H}$. If one defines $x_{1}=s_{1}, x_{2}=s_{1}^{*}, x_{3}=s_{2}$ and $x_{4}=s_{2}^{*}$, then it is easy to see, that $\mathcal{O}_{A}$ contains a dense sub-algebra $\mathcal{O}_{A}^{0}$, which is a free $\mathbb{C}$-algebra on four generators $x_{i}$ and three quadratic relations:

$$
\left\{\begin{array}{ccc}
x_{2} x_{1} & = & a_{11} x_{1} x_{2}+a_{12} x_{3} x_{4} \\
x_{4} x_{3} & = & a_{21} x_{1} x_{2}+a_{22} x_{3} x_{4} \\
1 & = & x_{1} x_{2}+x_{3} x_{4}
\end{array}\right.
$$

and an involution acting by the formula:

$$
x_{1}^{*}=x_{2}, \quad x_{3}^{*}=x_{4} .
$$

Notice, that equations (12) are invariant of this involution. 
Lemma $2\left(\mathcal{O}_{A}\right.$ as a crossed product) Let $\mathbb{A}$ be a stationary AF-algebra given by the inductive limit $\mathbb{Z}^{2} \stackrel{\mathrm{A}^{\mathrm{T}}}{\longrightarrow} \mathbb{Z}^{2} \stackrel{\mathrm{A}^{\mathrm{T}}}{\longrightarrow} \ldots$, where $A^{T}$ is the transpose of matrix A. Then

$$
\mathcal{O}_{A} \otimes \mathcal{K} \cong \mathbb{A} \rtimes_{\alpha} \mathbb{Z}
$$

where $\mathcal{K}$ is the $C^{*}$-algebra of compact operators and $\alpha$ the shift automorphism of $\mathbb{A}$. In particular, $\mathbb{A}$ is a sub-C $C^{*}$-algebra of the stabilization of the CuntzKrieger algebra $\mathcal{O}_{A} \otimes \mathcal{K}$.

Proof. We refer the reader to [Effros 1980] [4, Chapter 6 for the definition of stationary AF-algebra, shift automorphism, etc.; see [Blackadar 1986] [1], Chapter $\mathrm{V}$ for the definition of crossed product $C^{*}$-algebras. For a proof of lemma 2, see [Blackadar 1986] [1], Exercise 10.11.9.

\section{Proof of theorem 1}

Let the ground field be complex numbers, i.e. $k=\mathbb{C}$. We shall split the proof in a series of lemmas starting with the following elementary

Lemma 3 The ideal of free algebra $\mathbb{C}\left\langle x_{1}, x_{2}, x_{3}, x_{4}\right\rangle$ generated by equations (3) is invariant under involution (13), if and only if, $\bar{\alpha}=\alpha, \beta=1$ and $\gamma=-1$.

Proof. (i) Let us consider the first two equations (3); this pair is invariant of involution (13). Indeed, by the rules of composition for an involution

$$
\left\{\begin{array}{l}
\left(x_{1} x_{2}\right)^{*}=x_{2}^{*} x_{1}^{*}=x_{1} x_{2}, \\
\left(x_{2} x_{1}\right)^{*}=x_{1}^{*} x_{2}^{*}=x_{2} x_{1}, \\
\left(x_{3} x_{4}\right)^{*}=x_{4}^{*} x_{3}^{*}=x_{3} x_{4}, \\
\left(x_{4} x_{3}\right)^{*}=x_{3}^{*} x_{4}^{*}=x_{4} x_{3} .
\end{array}\right.
$$

Since $\alpha^{*}=\bar{\alpha}=\alpha$, the first two equation (3) remain invariant of involution (13).

(ii) Let us consider the middle pair of equations (3); by the rules of composition for an involution

$$
\left\{\begin{array}{l}
\left(x_{1} x_{3}\right)^{*}=x_{3}^{*} x_{1}^{*}=x_{4} x_{2}, \\
\left(x_{3} x_{1}\right)^{*}=x_{1}^{*} x_{3}^{*}=x_{2} x_{4}, \\
\left(x_{2} x_{4}\right)^{*}=x_{4}^{*} x_{2}^{*}=x_{3} x_{1}, \\
\left(x_{4} x_{2}\right)^{*}=x_{2}^{*} x_{4}^{*}=x_{1} x_{3} .
\end{array}\right.
$$


One can apply the involution to the first equation $x_{1} x_{3}-x_{3} x_{1}=\beta\left(x_{4} x_{2}+\right.$ $\left.x_{2} x_{4}\right)$; then one gets $x_{4} x_{2}-x_{2} x_{4}=\bar{\beta}\left(x_{1} x_{3}+x_{3} x_{1}\right)$. But the second equation says that $x_{1} x_{3}+x_{3} x_{1}=x_{4} x_{2}-x_{2} x_{4}$; the last two equations are compatible if and only if $\bar{\beta}=1$. Thus, $\beta=1$.

The second equation in involution writes as $x_{4} x_{2}+x_{2} x_{4}=x_{1} x_{3}-x_{3} x_{1}$; the last equation coincides with the first equation for $\beta=1$.

Therefore, $\beta=1$ is necessary and sufficient for invariance of the middle pair of equations (3) with respect to involution (13).

(iii) Let us consider the last pair of equations (3); by the rules of composition for an involution

$$
\left\{\begin{array}{l}
\left(x_{1} x_{4}\right)^{*}=x_{4}^{*} x_{1}^{*}=x_{3} x_{2}, \\
\left(x_{4} x_{1}\right)^{*}=x_{1}^{*} x_{4}^{*}=x_{2} x_{3}, \\
\left(x_{2} x_{3}\right)^{*}=x_{3}^{*} x_{2}^{*}=x_{4} x_{1} \\
\left(x_{3} x_{2}\right)^{*}=x_{2}^{*} x_{3}^{*}=x_{1} x_{4}
\end{array}\right.
$$

One can apply the involution to the first equation $x_{1} x_{4}-x_{4} x_{1}=\gamma\left(x_{2} x_{3}+\right.$ $\left.x_{3} x_{2}\right)$; then one gets $x_{3} x_{2}-x_{2} x_{3}=\bar{\gamma}\left(x_{4} x_{1}+x_{1} x_{4}\right)$. But the second equation says that $x_{1} x_{4}+x_{4} x_{1}=x_{2} x_{3}-x_{3} x_{2}$; the last two equations are compatible if and only if $\bar{\gamma}=-1$. Thus, $\gamma=-1$.

The second equation in involution writes as $x_{3} x_{2}+x_{2} x_{3}=x_{4} x_{1}-x_{1} x_{4}$; the last equation coincides with the first equation for $\gamma=-1$.

Therefore, $\gamma=-1$ is necessary and sufficient for invariance of the last pair of equations (3) with respect to involution (13).

(iv) It remains to verify that condition $\alpha+\beta+\gamma+\alpha \beta \gamma=0$ is satisfied by $\beta=1$ and $\gamma=-1$ for any $\alpha \in k$. Lemma 3 follows.

Remark 1 The Sklyanin algebra $\mathfrak{S}_{\alpha, 1,-1}(\mathbb{C})$ with $\alpha \in \mathbb{R}$ is a $*$-algebra with the involution $x_{1}^{*}=x_{2}$ and $x_{3}^{*}=x_{4}$.

Lemma 4 The first pair of equations (3):

$$
\left\{\begin{array}{ccc}
x_{1} x_{2}-x_{2} x_{1} & = & \alpha\left(x_{3} x_{4}+x_{4} x_{3}\right) \\
x_{1} x_{2}+x_{2} x_{1} & = & x_{3} x_{4}-x_{4} x_{3}
\end{array}\right.
$$

is equivalent to the pair:

$$
\left\{\begin{array}{l}
x_{2} x_{1}=\frac{1+\alpha}{1-\alpha} x_{1} x_{2}-\frac{2 \alpha}{1-\alpha} x_{3} x_{4} \\
x_{4} x_{3}=-\frac{2}{1-\alpha} x_{1} x_{2}+\frac{1+\alpha}{1-\alpha} x_{3} x_{4}
\end{array}\right.
$$


Proof. Let us isolate $x_{2} x_{1}$ and $x_{4} x_{3}$ in equations (18); for that, we shall write (18) in the form

$$
\left\{\begin{array}{c}
x_{2} x_{1}+\alpha x_{4} x_{3}=x_{1} x_{2}-\alpha x_{3} x_{4} \\
x_{2} x_{1}+x_{4} x_{3}=-x_{1} x_{2}+x_{3} x_{4}
\end{array}\right.
$$

Consider (20) as a linear system of equations relatively $x_{2} x_{1}$ and $x_{4} x_{3}$; since $\alpha \neq 1$, it has a unique solution

$$
\left\{\begin{array}{l}
x_{2} x_{1}=\frac{1}{1-\alpha}\left|\begin{array}{cc}
x_{1} x_{2}-\alpha x_{3} x_{4} & \alpha \\
-x_{1} x_{2}+x_{3} x_{4} & 1
\end{array}\right|=\frac{1+\alpha}{1-\alpha} x_{1} x_{2}-\frac{2 \alpha}{1-\alpha} x_{3} x_{4}, \\
x_{4} x_{3}=\frac{1}{1-\alpha}\left|\begin{array}{cc}
1 & x_{1} x_{2}-\alpha x_{3} x_{4} \\
1 & -x_{1} x_{2}+x_{3} x_{4}
\end{array}\right|=-\frac{2}{1-\alpha} x_{1} x_{2}+\frac{1+\alpha}{1-\alpha} x_{3} x_{4} .
\end{array}\right.
$$

Lemma 4 follows.

Remark 2 In new variables $\left(x_{2} x_{1}\right)^{\prime}=(1-\alpha) x_{2} x_{1}$ and $\left(x_{4} x_{3}\right)^{\prime}=(1-\alpha) x_{4} x_{3}$ the system of equations (19) can be written in the form:

$$
\left\{\begin{array}{l}
x_{2} x_{1}=(1+\alpha) x_{1} x_{2}-2 \alpha x_{3} x_{4}, \\
x_{4} x_{3}=-2 x_{1} x_{2}+(1+\alpha) x_{3} x_{4}
\end{array}\right.
$$

Lemma 5 (Main lemma) Let $\alpha_{k}=\sum_{i=1}^{k} b_{i} p^{i}$ be a rational integer and $A=\left(\begin{array}{cc}1+\alpha_{k} & -2 \alpha_{k} \\ -2 & 1+\alpha_{k}\end{array}\right)$. Suppose $M_{\alpha_{k}}$ is the UHF-algebra defined in Section 1 and $\mathbb{A}$ an AF-algebra introduced in lemma 2. Let $I_{0}$ be the (two-sided) ideal of the Sklyanin $*$-algebra $\mathfrak{S}_{\alpha_{k}, 1,-1}(\mathbb{C})$ generated by relation $x_{1} x_{2}+x_{3} x_{4}=1$ and $J_{0}$ the ideal of $\mathcal{O}_{A}^{0}$ generated by relations $x_{4} x_{2}-x_{1} x_{3}=x_{3} x_{1}+x_{2} x_{4}=$ $x_{4} x_{1}-x_{2} x_{3}=x_{3} x_{2}+x_{1} x_{4}=0$. Then there exists $a *$-isomorphism

$$
\mathfrak{S}_{\alpha_{k}, 1,-1}(\mathbb{C}) / I_{0} \cong \mathcal{O}_{A}^{0} / J_{0}
$$

where

$$
\overline{\mathcal{O}_{A}^{0}} \cong \mathcal{O}_{A} \quad \text { and } \quad \mathcal{O}_{A} \otimes \mathcal{K} \supset \mathbb{A} \supset M_{\alpha_{k}}
$$

are inclusions of the $C^{*}$-algebras.

Proof. Recall that there exists a dense inclusion $\mathbb{Z} \hookrightarrow \mathbf{Z}_{p}$ given by formula $\alpha_{k} \mapsto \sum_{i=1}^{k} b_{i} p^{i}$, where $0 \leq b_{i} \leq p-1$ are integer numbers, see e.g. [Gouvêa 1993] [6], Proposition 3.3.4 (ii); we shall use the inclusion to identify $p$-adic 
integer $\sum_{i=1}^{k} b_{i} p^{i}$ with the corresponding rational integer $\alpha_{k}$. Because $\alpha_{k} \in \mathbb{R}$, one gets a Sklyanin $*$-algebra $\mathfrak{S}_{\alpha_{k}, 1,-1}(\mathbb{C})$ with the involution $x_{1}^{*}=x_{2}$ and $x_{3}^{*}=x_{4}$, see remark 1]. Notice that the inclusion $\mathbb{Z} \hookrightarrow \mathbf{Z}_{p}$ induces a $*$ isomorphism between the following Sklyanin algebras:

$$
\mathfrak{S}_{\alpha_{k}, 1,-1}(\mathbb{C}) \cong \mathfrak{S}_{\sum_{i=1}^{k} b_{i} p^{i}, 1,-1}\left(\mathbf{Q}_{p}\right) .
$$

However, the norm convergence in the above families of Sklyanin algebras is different; in what follows, we deal with the Sklyanin algebras $\mathfrak{S}_{\alpha_{k}, 1,-1}(\mathbb{C})$ endowed with the usual archimedian norm.

Part I. To prove formula (23), one compares relations (3) defining the Sklyanin algebra $\mathfrak{S}_{\alpha, \beta, \gamma}(k)$ with relations (12) defining dense sub-algebra $\mathcal{O}_{A}^{0}$ of the Cuntz-Krieger algebra $\mathcal{O}_{A}$. It is easy to see, that ideal $J_{0}$ is generated by the last four relations of system (3) corresponding to the case $\beta=-\gamma=1$. Likewise, ideal $I_{0}$ is generated by the last relation of system (12).

As for the first pair of relations of systems (3) and (12), they are identical after a substitution $a_{11}=a_{22}=1+\alpha_{k}, a_{12}=-2 \alpha_{k}$ and $a_{21}=-2$, see also lemma 4 and remark 2. Thus, one gets isomorphism (23), where matrix $A$ is given by the formula $A=\left(\begin{array}{cc}1+\alpha_{k} & -2 \alpha_{k} \\ -2 & 1+\alpha_{k}\end{array}\right)$.

Part II. One can prove inclusions (24) in the following steps.

(i) The isomorphism $\overline{\mathcal{O}_{A}^{0}} \cong \mathcal{O}_{A}$ follows from definition of the CuntzKrieger algebra as the norm-closure of algebra $\mathcal{O}_{A}^{0}$.

(ii) In view of lemma 2, there exists a sub- $C^{*}$-algebra $\mathbb{A} \subset \mathcal{O}_{A} \otimes \mathcal{K}$; the sub- $C^{*}$-algebra is the stationary AF-algebra (see [Effros 1980] [4], Chapter $6)$ given by the following inductive limit:

$$
\mathbb{Z}^{2}\left(\begin{array}{cc}
1+\alpha_{k} & -2 \\
-2 \alpha_{k} & 1+\alpha_{k}
\end{array}\right) \mathbb{Z}^{2}\left(\begin{array}{cc}
1+\alpha_{k} & -2 \\
-2 \alpha_{k} & 1+\alpha_{k}
\end{array}\right) \ldots
$$

Notice that since $\alpha_{k}$ are positive integers, matrix $A^{T}$ has two negative offdiagonal entries. However, since $\operatorname{tr}\left(A^{T}\right)>2$ there exists a matrix in the similarity class of $A^{T}$ all of whose entries are positive; the inductive limit (26) is invariant of the similarity class.

(iii) To establish inclusion $M_{\alpha_{k}} \subset \mathbb{A}$, let us calculate the dimension group of AF-algebra $\mathbb{A}$, see [Effros 1980] 4] for definition of such a group. It 
is known, that for stationary AF-algebra $\mathbb{A}$ the dimension group is orderisomorphic to $\mathbb{Z}\left[\frac{1}{\lambda_{A^{T}}}\right]$, where $\lambda_{A^{T}}$ is the maximal eigenvalue of matrix $A^{T}$. We encourage the reader to verify that

$$
\mathbb{Z}\left[\frac{1}{\lambda_{A^{T}}}\right]=\mathbb{Z}\left[\frac{1+\alpha_{k}+2 \sqrt{\alpha_{k}}}{\left(\alpha_{k}-1\right)^{2}}\right] .
$$

It follows from (27) that

$$
\mathbb{Z}\left[\frac{1}{\alpha_{k}-1}\right] \subset \mathbb{Z}\left[\frac{1}{\lambda_{A^{T}}}\right]
$$

is an inclusion of dimension groups. In view of corollary 1, one can replace $\alpha_{k}-1$ by $-\alpha_{k}$ in formula (28); therefore, one gets the inclusion:

$$
\mathbb{Z}\left[\frac{1}{\alpha_{k}}\right] \subset \mathbb{Z}\left[\frac{1}{\lambda_{A^{T}}}\right] .
$$

Because $\alpha_{k} \notin\{0 ; \pm 1\}$ (see Section 2.1), one concludes that $\mathbb{Z}\left[\frac{1}{\alpha_{k}}\right]$ is a dense abelian subgroup of the rational numbers $\mathbb{Q}$. It remains to notice that the dimension group $\mathbb{Z}\left[\frac{1}{\alpha_{k}}\right]$ is order-isomorphic to such of the UHF-algebra $M_{\alpha_{k}}$; see definition of $M_{\alpha_{k}}$ in Section 1. Thus inclusion (29) implies the inclusion $M_{\alpha_{k}} \subset \mathbb{A}$. Lemma 5 follows.

Theorem 1 follows from lemma 5 .

\section{Example}

We shall consider an example illustrating theorem 1. Let $p$ be a prime number and consider the $p$-adic integer of the form $q=p$; notice that in this case $b_{1}=1$ and $b_{2}=b_{3}=\ldots=0$. One gets therefore a supernatural number $\mathbf{n}(q)$ of the form $p^{\infty}$. The $\mathbf{n}(q)$ corresponds to a dense subgroup of $\mathbb{Q}$ of the form:

$$
\mathbb{Q}(\mathbf{n})=\mathbb{Z}\left[\frac{1}{p}\right] .
$$

It is easy to see, that the UHF-algebra corresponding to the Tate curve $\mathcal{E}_{p}=\mathbf{Q}_{p}^{*} / p^{\mathbb{Z}}$ has the form:

$$
M_{p^{\infty}}:=M_{p}(\mathbb{C}) \otimes M_{p}(\mathbb{C}) \otimes \ldots
$$


In virtue of theorem 1, the UHF-algebra $M_{p^{\infty}}$ is (a sub- $C^{*}$-algebra of the stable closure of infinite-dimensional representation of quotient ring of) the twisted homogeneous coordinate ring of the Tate curve $\mathcal{E}_{p}$. In particular, for $\mathcal{E}_{2}$ such a coordinate ring is the UHF-algebra $M_{2 \infty}$; the latter is known as a Canonical Anticommutation Relations $C^{*}$-algebra (the CAR or Fermion algebra) [Effros 1981] [4], p.13.

\section{References}

[1] B. Blackadar, K-Theory for Operator Algebras, MSRI Publications, Springer, 1986.

[2] A. Carey, M. Marcolli and A. Rennie, Modular index invariants of Mumford curves, in: Noncommutative geometry, arithmetic, and related topics. Proceedings of the 21st meeting of the Japan-U.S. Mathematics Institute (JAMI) held at Johns Hopkins University, Baltimore, MD, USA, March 23-26, 2009. Baltimore, MD: Johns Hopkins University Press. 31-73 (2011).

[3] J. Cuntz and W. Krieger, A class of $C^{*}$-algebras and topological Markov chains, Invent. Math. 56 (1980), 251-268.

[4] E. G. Effros, Dimensions and $C^{*}$-Algebras, in: Conf. Board of the Math. Sciences No.46, AMS (1981).

[5] J. G. Glimm, On a certain class of operator algebras, Trans. Amer. Math. Soc. 95 (1960), 318-340.

[6] F. O. Gouvêa, P-Adic Numbers, Springer, 1993.

[7] G. J. Murphy, $C^{*}$-Algebras and Operator Theory, Academic Press, 1990.

[8] M. Rørdam, F. Larsen and N. Laustsen, An introduction to $K$-theory for $C^{*}$-algebras. London Mathematical Society Student Texts, 49. Cambridge University Press, Cambridge, 2000.

[9] E. K. Sklyanin, Some algebraic structures connected to the YangBaxter equations, Functional Anal. Appl. 16 (1982), 27-34. 
[10] S. P. Smith and J. T. Stafford, Regularity of the four dimensional Sklyanin algebra, Compositio Math. 83 (1992), 259-289.

[11] J. T. Stafford and M. van den Bergh, Noncommutative curves and noncommutative surfaces, Bull. Amer. Math. Soc. 38 (2001), 171-216.

[12] J. T. Tate, The arithmetic of elliptic curves, Inventiones Math. 23 (1974), 179-206.

The Fields Institute for Research in Mathematical Sciences, Toronto, ON, Canada, E-MaIL: igor.v.nikolaev@gmail.com 